\title{
UTILIZAÇÃO DE MATRIZ DE RISCOS PARA ANÁLISE DE MICROACESSIBILIDADE EM UMA VIA ARTERIAL DE FORTALEZA
}

\author{
DE ABREU E TRÉZ, Juliana (1); \\ AUGUSTO E SÁ MAGALHÃES, Pedro (2); \\ DUTRA MONTENEGRO, Nadja G da S. (3) \\ (1) Universidade Federal do Ceará, Graduada \\ e-mail:julianatrez@gmail.com \\ (2) Universidade Federal do Ceará, Graduado \\ e-mail:pedromagalhaes.sa@hotmail.com \\ (3) Universidade Federal do Ceará, D.Sc \\ e-mail:nadja@det.ufc.br
}

\begin{abstract}
RESUMO
As discussões acerca de acessibilidade ganham, cada vez mais, destaque no cenário do planejamento urbano, sempre tendo como base o Desenho Universal. Contudo, na maioria das vezes, os projetos acabam não contemplando significativamente os pedestres, principalmente aqueles com deficiência, impedindo sua utilização do espaço urbano. Este artigo tem como objetivo analisar a acessibilidade das calçadas de uma avenida de Fortaleza para sugerir intervenções, no intuito de melhorar sua qualidade. A ferramenta a ser utilizada nesse estudo será a Matriz de Riscos, que consiste na relação entre a probabilidade de os riscos acontecerem e seu impacto.

Palavras chave: Microacessibilidade; Calçada; Matriz de Riscos.
\end{abstract}

\begin{abstract}
Discussions about accessibility are increasingly important in the urban planning scenario, with the aid of the Universal Design. However, in most cases, projects don't think about the pedestrians, specially the ones with disabilities, preventing them from using the urban area. This paper aims to analyse the accessibility of sidewalks in an avenue located in the city of Fortaleza to suggest solutions, with the intention of improve their quality. It will be possible using the Risk Matrix, that is a board that relates the probability of risks happen and their impact.
\end{abstract}

Keywords: Microaccessibility; Sidewalk; Risk Matrix.

\section{INTRODUÇÃO}

O ser humano está em constante movimento e se desloca com frequência de um lugar para o outro, sobretudo a pé, sendo esse o meio mais básico de deslocamento para todos nas cidades, independentemente de sua estrutura física, renda e oferta de transportes (VASCONCELLOS, 2001). Mesmo assim, há pessoas que encontram mais dificuldade nos deslocamentos, como idosos, gestantes, pessoas temporariamente acidentadas e aquelas com deficiência, que necessitam, muitas vezes, de cadeira de rodas e muletas. Dito isso, é 


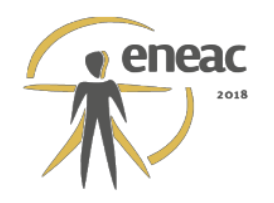

fácil perceber que os pedestres, sobretudo os que tem dificuldades de locomoção, precisam de um espaço urbano acessível, bem planejado, sinalizado e sem obstáculos, seguindo assim os preceitos do Desenho Universal.

Nos países em desenvolvimento há uma falta de qualidade das calçadas e de condições adequadas para cruzar as vias, sendo possível concluir que suas cidades não são construídas para os pedestres (VASCONCELLOS, 2001), chamando assim a atenção para um grande problema de acessibilidade, em termos estruturais e, sobretudo, de inclusão social daqueles com restrições de mobilidade. Faz-se necessário então um estudo da qualidade das calçadas e acessos para que sejam adotadas medidas que tragam maior conforto, segurança e autonomia para os pedestres, de acordo com as normas e leis vigentes. É muito importante também uma análise do que deve ser prioridade na correção, dependendo da urgência e do grau de perigo oferecido.

Diante do exposto, torna-se interessante a utilização de uma Matriz de Riscos, amplamente utilizada em gestão de empresas, voltada aqui para a microacessibilidade, adiante definida. O Departamento Nacional de Infraestrutura de Transportes (DNIT, 2013, p. 13) define a matriz de riscos como "um instrumento de gestão que, além de documentar as informações dos riscos, auxilia na comunicação e dá subsídio às demais etapas do gerenciamento de riscos, sendo importante aliada no gerenciamento de recursos", visto que os órgãos públicos dispõem de um orçamento que dificilmente supre todas as necessidades de imediato.

\section{FUNDAMENTAÇÃO TEÓRICA}

\subsection{Acessibilidade, Mobilidade e Desenho Universal}

A NBR 9050 da Associação Brasileira de Normas Técnicas (ABNT, 2015) e o Decreto no 5296 (BRASIL, 2004) definem acessibilidade como a possibilidade de utilização por pessoas com deficiência e mobilidade reduzida das edificações, do espaço e do mobiliário urbano, com conforto, segurança e autonomia.

Litman (2011) define acessibilidade dentro do contexto dos transportes, como sendo a capacidade de se utilizar bens e serviços, executar atividades e se chegar a destinos, sendo reflexo da habilidade de locomoção da pessoa e do padrão de uso do espaço. Ela é avaliada baseada no tempo e dinheiro exigidos e no desconforto e risco causados para alcançar um determinado objetivo, sendo relativamente difícil de ser mensurada por ser influenciada por diversos fatores.

Vasconcellos (2001), por sua vez, divide a acessibilidade em macroacessibilidade e microacessibilidade. A primeira aborda a facilidade de o indivíduo alcançar o local desejado, tendo relação com a estrutura das vias e dos transportes. Já a segunda se concentra na relação entre o pedestre e o percurso que ele faz, podendo ser positiva ou negativa, dependendo dos elementos que ele encontra nesse caminho.

A mobilidade pode ser entendida, de acordo com o Ministério das Cidades (BRASIL, 2006), pelo deslocamento de uma pessoa em virtude de suas condições físicas e econômicas. Esse conceito, quando especificamente relacionado à cidade, recebe o nome de mobilidade urbana e consiste, como dito na Lei no 12.587 (BRASIL, 2012), no deslocamento de pessoas e cargas no espaço urbano, podendo ser de forma não-motorizada, como a pé ou de bicicleta, ou motorizada, através de veículo particular ou transporte público.

$\mathrm{Na}$ prática, as definições de acessibilidade e mobilidade estão intimamente relacionadas, pois é necessário que o espaço urbano ofereça condições físicas adequadas para que a 


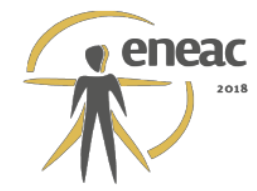

movimentação através dele seja feita com conforto e segurança por todas as pessoas, independentemente de suas limitações. Ou seja, é preciso acessibilidade para que aconteça a mobilidade com autonomia.

De acordo com Brasil (2004), o Desenho Universal é a concepção de espaços, artefatos e produtos com o objetivo de atender, de forma simultânea, a todas as pessoas, com diferentes características antropométricas e sensoriais, garantindo conforto, segurança e autonomia.

Um projeto que respeita os princípios do Desenho Universal deve ser realizado de modo integrado, de acordo com as necessidades de todos os indivíduos (AGUIAR, 2010). Cambiaghi (2012) ressalta que o conceito de Desenho Universal está ligado à diversidade da população, almejando uma igualdade na capacidade de acesso e utilização dos elementos do espaço urbano, enfatizando a inclusão social, de forma a incentivar a produção de ambientes que possuam elementos especiais para pessoas com deficiência e mobilidade reduzida.

A acessibilidade e a mobilidade estão diretamente ligadas ao conceito de Desenho Universal, já que o principal objetivo deste é possibilitar o acesso aos bens e serviços para o maior número possível de pessoas, independentemente de tamanho, idade, gênero e mobilidade. Pode-se alcançar esse objetivo com projetos que respeitem a diversidade física e sensorial dos usuários e preservem sua autonomia.

A Figura 1 apresenta uma pirâmide que sugere o grau de dificuldade encontrado por tipo de deficiência física. Assim, quanto mais no topo, maiores as dificuldades de deslocamento e, quanto mais observados os critérios para o pior tipo de restrição, melhores e mais acessíveis serão os ambientes criados.

Figura 1 - A pirâmide do Desenho Universal

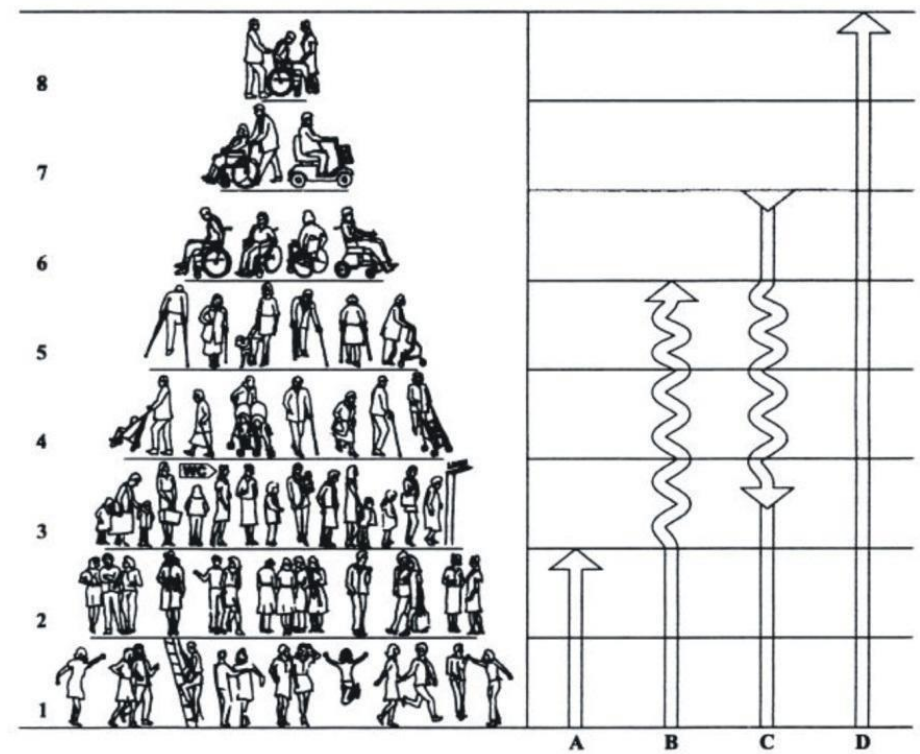

Fonte: GOLDSMITH (2001) apud CAMPÊLO (2011).

\subsection{Matriz de Riscos}

De acordo com Scofano et al (2013), um risco pode ser definido como fatores incertos às expectativas, algo que atua incessantemente sobre os objetivos e metas, intervindo no 


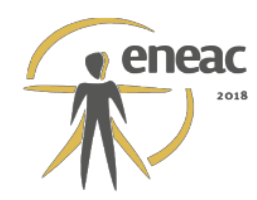

ambiente e causando prejuízos. Para antecipar os problemas e encontrar soluções para resolvê-los, caso eles sejam inevitáveis, efetua-se uma análise de riscos, que é o processo de caracterizar, gerenciar e informar sobre a existência e magnitude das perdas potenciais do projeto (MODARRES, 2006). Essa análise pode ser feita de várias formas, e uma delas é através de uma Matriz de Riscos.

Uma Matriz de Riscos é um método utilizado no gerenciamento, o qual identifica o que é mais crítico em uma determinada situação e oferece uma metodologia para avaliar os potenciais impactos dos problemas encontrados e de suas soluções (GARVEY, LANSDOWNE; 1998). Ela é elaborada através da relação entre os valores de impacto e a probabilidade mensurados para cada problema (risco). Visualmente, ela é um quadro com " $m$ " linhas e " $n$ " colunas, onde $m$ e $n$ variam de acordo com o detalhamento das escalas adotadas para o impacto e a probabilidade.

Conforme explicado, uma calçada tem como objetivo o trânsito livre e seguro do pedestre, tenha ele restrições de mobilidade ou não. Portanto, pensar no conceito de risco em microacessibilidade remete à diminuição ou ao impedimento da locomoção do pedestre em calçadas. Assim, os riscos identificados para montagem da Matriz foram aqueles presentes no passeio, que dificultam o deslocamento acessível, podendo até prejudicar a integridade física de quem se arrisca.

\subsection{Critérios para Calçadas Acessíveis}

De acordo com o Código de Trânsito Brasileiro (BRASIL, 1997), calçada é "parte da via, normalmente segregada e em nível diferente, não destinada à circulação de veículos, reservada ao trânsito de pedestres e, quando possível, à implantação de mobiliário, sinalização, vegetação, placas de sinalização e outros fins". As calçadas possibilitam a utilização do mobiliário urbano e conectam o espaço público ao privado, permitindo o acesso às edificações, e, para que isso seja possível, é preciso que elas tenham largura de passagem adequada, sem inclinações exageradas ou irregularidades no piso e façam parte de rotas contínuas que também incluem itens como faixas de pedestres e guias rebaixadas, sempre seguindo os preceitos do Desenho Universal.

Segundo ABNT (2015), a calçada pode ser subdividida em três faixas: faixa de serviço, faixa livre e faixa de acesso. A faixa de serviço tem como função acomodar o mobiliário urbano, a faixa de acesso é aquela localizada na entrada dos edifícios e serve de apoio para a projeção de marquises, toldos e rampas de acesso, e a faixa livre é a área da calçada destinada exclusivamente ao trânsito de pedestres e a que precisa de uma maior preocupação. Essa faixa deve ser livre de qualquer obstáculo e ter, no mínimo, 1,20m de largura, além de uma inclinação transversal máxima de $3 \%$ e um piso plano, uniforme e antiderrapante para ser considerada segura e acessível para as pessoas com deficiência e mobilidade reduzida.

É necessário também que a calçada acomode equipamento urbano adequado de travessia em sua esquina (as guias rebaixadas) para que a pessoa em cadeira de rodas ou com algum tipo de problema motor possa atravessar as vias sem riscos à sua integridade física, fortalecendo a continuidade no trajeto entre as calçadas, sem esquecer do piso tátil direcional ao final da guia para as pessoas com deficiência visual. 


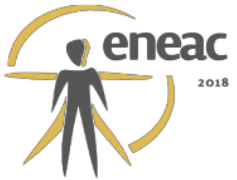

\section{METODOLOGIA}

\subsection{Escolha do Local}

O local escolhido para análise foi a Avenida Desembargador Moreira, em Fortaleza, por ser um importante corredor localizado em uma das regiões mais ricas comercialmente, devido à grande concentração de estabelecimentos comerciais, bancos, restaurantes etc., o que atrai um intenso fluxo de pessoas a pé. Foram escolhidos 16 quarteirões ao logo do trecho.

\subsection{Determinação dos critérios de impacto e probabilidade e montagem da Matriz}

Para elaboração da Matriz, primeiramente foi preciso determinar as escalas de impacto e probabilidade utilizadas para o estudo. Garvey e Lansdowne (1998) sugerem, em seu trabalho, 5 níveis para cada escala, sendo essa a quantidade de graus adotada, para posterior classificação dos riscos após o levantamento. As Tabelas 1 e 2 mostram as escalas de impacto e probabilidade e as descrições que foram consideradas para cada grau.

Tabela 1 - Escala de impacto

\begin{tabular}{cc}
\hline Descrição & Grau \\
\hline Não impede o deslocamento & 1 \\
Impede pouco o deslocamento & 2 \\
\hline Impede o deslocamento moderadamente; requer certo esforço & 3 \\
mpede o deslocamento em um grau significativo (necessita auxílio) & 4 \\
\hline Impede completamente o deslocamento, mesmo com auxílio & 5 \\
\hline
\end{tabular}

Fonte: Os autores.

Tabela 2 - Escala de probabilidade

\begin{tabular}{cc}
\hline Descrição & Grau \\
\hline Muito baixa (0 a 20\%) & 1 \\
Baixa (20 a 40\%) & 2 \\
\hline Intermediária (40 a 60\%) & 3 \\
Alta (60 a 80\%) & 4 \\
Muito alta (80 a 100\%) & 5 \\
\hline
\end{tabular}

Fonte: Os autores. 


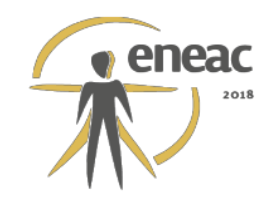

Após a definição das escalas, foi preciso quantificar os graus de impacto que seriam considerados para cada risco. A quantificação foi feita através de um questionário elaborado na página eletrônica Google Forms e divulgado para pessoas que precisam de cadeira de rodas, com uma lista dos riscos que foram determinados para observação, com opções de 1 a 5 correspondentes a cada grau de impacto na escala. Esse questionário foi baseado em Likert, uma abordagem que emite um grau de concordância para mensurar elementos de acordo com o posicionamento do respondente de forma qualitativa (SILVA JÚNIOR. COSTA; 2014).

A probabilidade foi mensurada como a relação entre a quantidade de quarteirões/lotes que apresentou o risco e o total de quarteirões/lotes. Dessa forma, assumiu-se que, quanto maior a repetição daquele risco, maior a dificuldade que a pessoa encontra para concluir seu trajeto.

A Matriz de Riscos será apresentada como uma Tabela, com o auxílio de cores para melhor identificação. Os riscos serão organizados em ordem decrescente, de acordo com os graus de impacto e probabilidade encontrados para cada. A Tabela 3 mostra um exemplo dessa organização.

Tabela 3 - Exemplo de Matriz de Risco

\begin{tabular}{c|cc}
\hline Risco & Impacto & Probabilidade \\
\hline A & 5 & 5 \\
B & 5 & 4 \\
C & 4 & 3 \\
D & 5 & 2 \\
E & 3 & 1 \\
\hline
\end{tabular}

Fonte: Os autores.

\subsection{Elaboração dos formulários e levantamento em campo}

Para o levantamento em campo, foram elaborados três formulários, sendo um para Calçadas, um para Esquinas e um para Mobiliário Urbano.

O levantamento foi feito com o auxílio dos formulários para anotações, trena métrica para medir as larguras livres e os espaços deixados por mobiliário urbano, uma câmera de celular para registrar as irregularidades encontradas e um aplicativo para celular para medição do ângulo de inclinação transversal do passeio.

Teoricamente, a calçada precisa ser contínua e com o mesmo padrão ao longo do quarteirão, mas, em Fortaleza, essa prática não observada, ficando cada dono de lote responsável pela manutenção de sua calçada. Essa situação foi observada no levantamento, pois havia calçadas que tinham mais de um estabelecimento lado a lado, surgindo assim a necessidade de aplicar os formulários de Calçada e Mobiliário Urbano em cada lote separadamente. 


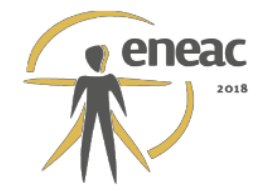

\section{RESULTADOS E DISCUSSÕES}

Para entendimento do que foi obtido através da metodologia apresentada, são apresentadas adiante partes dos resultados de impacto, probabilidade e da Matriz de Risco final.

Em relação à quantificação do grau de impacto individual, determinou-se que a alternativa mais respondida para cada problema seria a escolhida. A Tabela 4 lista o resumo dos graus de impacto determinados através desse método.

Tabela 4 - Graus de impacto

\begin{tabular}{lc}
\hline \multicolumn{1}{c}{ Risco } & $\begin{array}{c}\text { Grau de } \\
\text { impacto }\end{array}$ \\
\hline Inclinação transversal do passeio > 3\% & 5 \\
Piso irregular & 4 \\
Rampa de garagem & 5 \\
Ausência de rebaixamento calçada (guia) & 5 \\
Guia desnivelada c/meio-fio & 4 \\
\hline
\end{tabular}

Fonte: Os autores.

Observando-se os graus de impacto encontrados, nota-se uma repetição dos graus 4 e 5, o que já é, por si só, preocupante, visto que as pessoas que responderam consideram essas barreiras um grande complicador no trajeto.

A probabilidade foi calculada efetuando a contagem da quantidade de repetições e dividindo essa quantidade pelo número total de análises, dependendo do formulário (lotes ou ruas transversais). A Tabela 5 exibe um resumo dos resultados das planilhas, listando os riscos e seus valores de probabilidade tanto em porcentagem, quanto dentro da escala.

Tabela 5 - Graus de probabilidade

\begin{tabular}{lcc}
\hline \multicolumn{1}{c}{ Risco } & $\begin{array}{c}\text { Probabilidade } \\
(\%)\end{array}$ & Grau \\
\hline Inclinação transversal do passeio > 3\% & 56,14 & 3 \\
\hline Piso irregular & 66,67 & 4 \\
\hline Rampa de garagem & 19,30 & 1 \\
\hline Ausência de rebaixamento calçada (guia) & 78,13 & 4 \\
\hline Guia desnivelada c/meio-fio & 90,63 & 5 \\
\hline
\end{tabular}

Fonte: Os autores. 


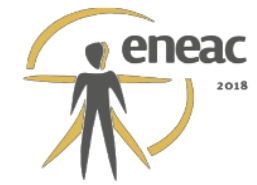

Os critérios mais problemáticos dentre todos os observados foram os que dizem respeito à guia rebaixada nas esquinas. Há uma carência muito grande desse equipamento nas ruas transversais do trecho, o que caracteriza descontinuidade, pois a pessoa não consegue atravessar a rua em segurança para continuar seu trajeto. A probabilidade encontrada para o rebaixamento de calçada desnivelado é maior ainda que a relacionada à sua ausência, o que preocupa, pois mostra que, mesmo com guias rebaixadas dos dois lados da rua, há uma dificuldade em sua utilização devido a instalações incorretas. Todavia, o mais frequente é a presença do rebaixamento em apenas um lado ou nenhum, já que muitas vezes são os próprios donos dos estabelecimentos de esquina que rebaixam suas calçadas.

Os riscos foram quantificados através da multiplicação entre seus graus de impacto e probabilidade, retornando um valor numérico, aqui chamado de Fator de Risco. Esse fator possibilita a elaboração de um ranking que apresenta as irregularidades encontradas no levantamento, auxiliando no mapeamento dos problemas, que necessitam ser resolvidos pelo poder público com maior urgência, os que apresentam os maiores valores. A Tabela 6 apresenta os riscos com seus respectivos graus de impacto e probabilidade e seus fatores de risco.

Tabela 6 - Ranking dos riscos da Av. Desembargador Moreira

\begin{tabular}{cccc}
\hline Risco & Impacto & Probab. & $\begin{array}{c}\text { Fator } \\
\text { de } \\
\text { Risco }\end{array}$ \\
\hline $\begin{array}{c}\text { Ausência de rebaixamento calçada (guia) } \\
\text { Guia desnivelada c/meio-fio }\end{array}$ & 5 & 4 & 20 \\
Piso irregular & 4 & 5 & 20 \\
Inclinação transversal > 3\% & 4 & 4 & 16 \\
Rampa de garagem & 5 & 3 & 15 \\
\hline
\end{tabular}

Fonte: Os autores.

A interpretação que pode ser feita observando-se a Matriz é que a pessoa em cadeira de rodas não consegue efetuar um trajeto contínuo ao longo da Avenida, pois não há uma infraestrutura para atravessar as ruas entre os quarteirões, impedindo a utilização plena do espaço urbano e seus serviços.

Encontrados os Fatores de Risco para cada problema, foi possível utilizá-los para obter um valor absoluto de risco para cada quarteirão, através da ponderação desses Fatores. $O$ recurso de cores foi utilizado para facilitar a diferenciação entre quarteirões que apresentaram valores absolutos de risco baixos (verde), médios (amarelo) e altos (laranja e vermelho), como mostrado no mapa temático da Figura 2. 
Figura 2 - Mapa temático com os Valores de Risco por quarteirão

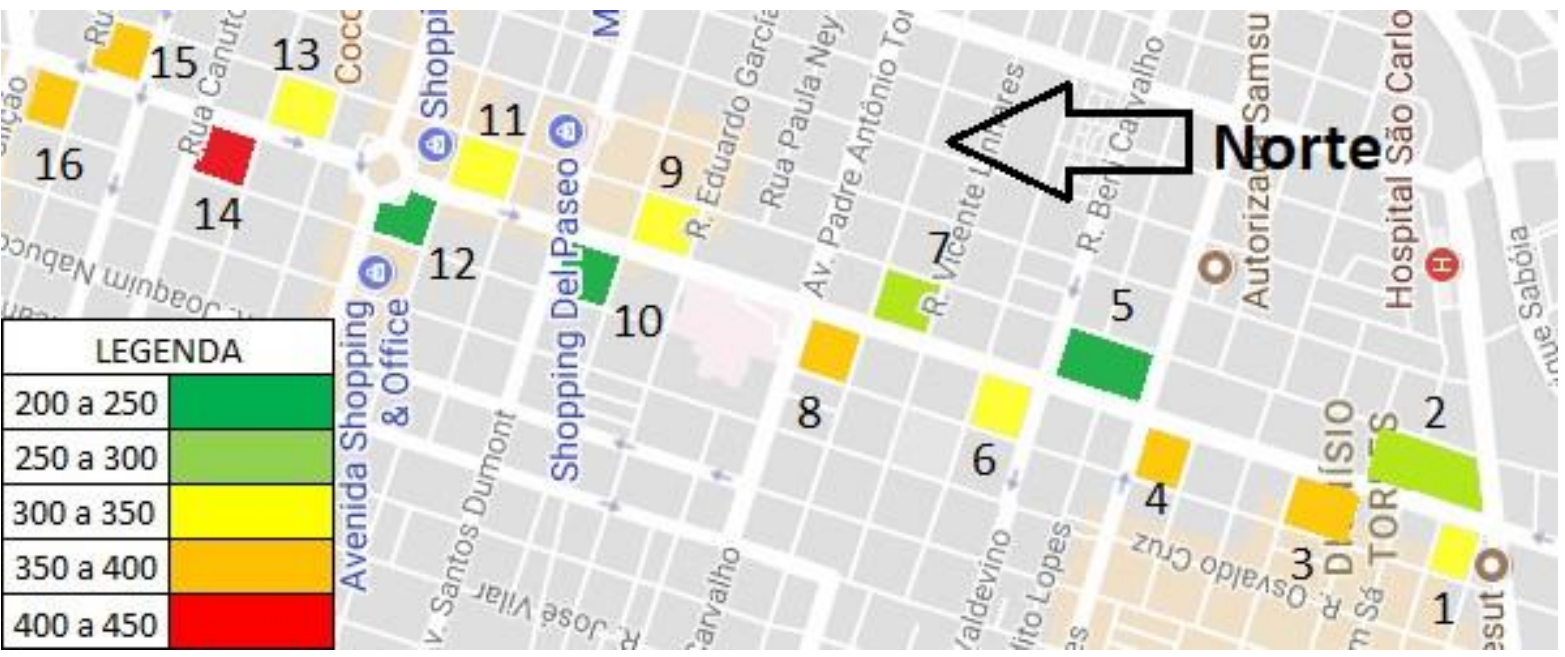

Fonte: Os autores.

De acordo com os cálculos feitos e a apresentação no mapa temático, o quarteirão mais problemático do trecho é o de número 14, que tem vários estabelecimentos comerciais com calçadas de inclinação transversal maior que 3\% devido a vagas para carros e rampas de garagem, além de irregularidades do piso.

A grande maioria dos quarteirões apresentou cor amarela, o que mostra que há irregularidades, mas não em todos os aspectos. Os quarteirões considerados menos problemáticos foram os de número 5,10 e 12 , sendo que os de número 10 e 12 apresentaram bons resultados, principalmente em relação às suas esquinas, pois foram feitas intervenções recentes de mobilidade urbana nas avenidas onde eles se localizam.

\section{CONSIDERAÇÕES FINAIS}

Após todas as etapas, concluiu-se que o estudo foi bem-sucedido, pois foi possível determinar os problemas (ou riscos) e sua intensidade em uma avenida de Fortaleza, com a criação de escalas de impacto e probabilidade e o levantamento em campo, além de ter sido feita a organização desses riscos em um ranking para averiguação dos problemas que precisariam ser prioridade no caso de intervenções para tornar o trecho seguro e acessível para a pessoa em cadeira de rodas, e da obtenção dos valores absolutos por quarteirão.

O estudo confirmou também que as calçadas da Avenida Desembargador Moreira apresentam vários padrões e uma infraestrutura precária e sem manutenção, com trechos completamente problemáticos em todos - ou quase todos - os aspectos observados

Esses fatores abrem precedentes para uma reflexão acerca da necessidade da aplicação de uma legislação que exija uma padronização das calçadas, pois essa falta de uniformidade é o maior problema quando se fala de acessibilidade em calçadas. Existe em Fortaleza o Código de Obras e Posturas, que foi reformulado em 2017, que determina critérios para execução de obras públicas e particulares, podendo auxiliar nessa uniformização da calçada se houver compromisso de uma fiscalização mais rigorosa. 


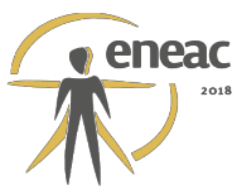

\section{REFERÊNCIAS BIBLIOGRÁFICAS}

AGUIAR, Fabiola de Oliveira. Acessibilidade Relativa dos Espaços Urbanos para Pedestres com Restrições de Mobilidade. 2010. 190 f. Tese (Doutorado em Engenharia de Transportes) - Escola de Engenharia de São Carlos, Universidade de São Paulo, São Carlos, 2010.

ASSOCIAÇÃO BRASILEIRA DE NORMAS TÉCNICAS. NBR 9050: Acessibilidade a edificações, mobiliário, espaços e equipamentos urbanos. 3 ed. Rio de Janeiro, 2015. 148 p.

BRASIL. Decreto $n^{\circ}$ 5.296, de 2 de dezembro de 2004, que regulamenta a Lei $n^{\circ} 10.098$, de 19 de dezembro de 2000, que estabelece normas gerais e critérios básicos para a promoção da acessibilidade das pessoas portadoras de deficiência ou com mobilidade reduzida, e dá outras providências. Diário Oficial [da] República Federativa do Brasil, Brasília, DF, 03 dez. 2004.

BRASIL. Lei no 9.503, de 23 de setembro de 1997, que institui o Código de Trânsito Brasileiro. Diário Oficial [da] República Federativa do Brasil, Brasília, DF

BRASIL. Lei no 12.587, de 3 de janeiro de 2012, que institui as diretrizes da Política Nacional de Mobilidade Urbana. Diário Oficial [da] República Federativa do Brasil, Brasília, DF, 04 jan. 2012.

BRASIL. Secretaria Nacional de Transporte e de Mobilidade Urbana. Caderno 2 - Construindo a cidade acessível. Ministério das Cidades, Brasília, 2006. (Brasil Acessível).

CAMBIAGHI, Silvana. Desenho Universal: Métodos e técnicas para arquitetos e urbanistas. São Paulo: Editora Senac, 2007. 269 p.

CAMPÊLO, Ana Elisa Pinheiro. Proposição de modelo para escolha de rotas urbanas acessíveis considerando-se os critérios de microacessibilidade para as pessoas com deficiência física motora. 141 f. Dissertação (Mestrado) - Curso de Engenharia Civil, Departamento de Engenharia de Transportes, Universidade Federal do Ceará, Fortaleza, 2011.

DEPARTAMENTO NACIONAL DE INFRAESTRUTURA DE TRANSPORTES. Guia de Gerenciamento de Riscos de Obras Rodoviárias - Fundamentos. Brasília, 2013.

GARVEY, Paul R.; LANSDOWNE, Zachary F. Risk Matrix: an approach for identifying, assessing and ranking program risks. Air Force Journal Of Logistics, p. 16-19, 1998.

LITMAN, Todd. Measuring Transportation: traffic, mobility and accessibility. Victoria Transport Policy Institute (VTPI). Victoria, AUS, 2011. Disponível em: <http://www.vtpi.org/measure.pdf>. Acesso em: 24 maio 2017.

MODARRES, Mohammad. Risk analysis in Engineering: techniques, tools and trends. Boca Ratón: CRC, 2006. 391 p.

SCOFANO, C. R. F., ABRAHAM, E. F., SILVA, L. S., TEIXEIRA, M. A. Gestão De Risco Em Projetos: Análise Das Etapas Do PMI-PMBOK (Project Management Institute). In: XI Congresso Online de Administração, 2013, Brasília. Anais do XI Congresso Online de Administração, 2013.

SILVA JUNIOR, Severino Domingos da; COSTA, Francisco José da. Mensuração e escalas de verificação: uma análise comparativa das escalas de Likert e Phrase Completion. In: XVII Seminários em Administração. (Anais). São Paulo: FEA - USP, 2014. p. 1 - 16.

VASCONCELLOS, Eduardo A. Transporte urbano nos países em desenvolvimento: reflexões e propostas. 3. ed. São Paulo: Annablume, 2001. 282 p. 